\title{
Skeletal characterization in a patient with Hajdu-Cheney syndrome undergoing total knee arthroplasty
}

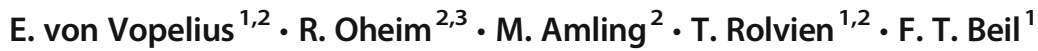

Received: 2 February 2021 / Accepted: 5 March 2021 / Published online: 19 March 2021

(C) The Author(s) 2021

\begin{abstract}
Hajdu-Cheney syndrome (HCS) is a rare genetic connective tissue disorder caused by gain-of-function mutations in the NOTCH2 gene. We report a 38-year-old male HCS patient with a history of multiple pathologic fractures, poor bone stock under intermittent antiresorptive therapy, and secondary osteoarthritis $(\mathrm{OA})$ of the knee, in which we successfully performed total knee arthroplasty (TKA). Next to a detailed skeletal assessment including laboratory bone metabolism markers, dual energy X-ray absorptiometry (DXA), and high-resolution peripheral quantitative computed tomography (HR-pQCT), undecalcified histologic and histomorphometric analysis was performed on intraoperatively obtained tibial cut sections. This multiscale assessment revealed a severe, combined trabecular-cortical microarchitectural deterioration, increased bone turnover indices, and advanced cartilage degeneration, thus demonstrating the crucial role of Notch2 in skeletal and cartilage homeostasis, which is in line with the findings of previous mouse models.
\end{abstract}

Keywords Hajdu-Cheney syndrome $\cdot$ Histomorphometry $\cdot$ NOTCH2 $\cdot$ Osteoarthritis $\cdot$ Osteoporosis $\cdot$ Total knee arthroplasty

\section{Introduction}

Hajdu-Cheney syndrome (HCS) is a rare genetic connective tissue disorder caused by heterozygous gain-of-function mutations of the $\mathrm{NOTCH} 2$ gene, resulting in premature translational termination. Since the deleted domain is required for protein degradation, these mutations result in Notch2 accumulation and increased downstream signaling [1, 2]. In skeletal homeostasis, Notch2 hyperactivity expresses constant inhibition of endochondral ossification (e.g., early skeletal growth, lifelong fracture healing) and stimulation of osteoclastogenesis, leading

T. Rolvien

t.rolvien@uke.de

$\triangle$ F. T. Beil

ft.beil@uke.de

$1 \quad$ Division of Orthopaedics, Department of Trauma and Orthopaedic Surgery, University Medical Center Hamburg-Eppendorf, Martinistraße 52, 20246 Hamburg, Germany

2 Department of Osteology and Biomechanics, University Medical Center Hamburg-Eppendorf, Hamburg, Germany

3 National Bone Board, Martin Zeitz Center for Rare Diseases, University Medical Center Hamburg-Eppendorf, Hamburg, Germany to skeletal developmental disorders and severe early-onset osteoporosis (EOOP) [3]. As previously reported, Notch2 serves as a major signaling pathway not only in skeletal but also in joint homeostasis [4]. In this regard, increased Notch signaling has been discussed in the context of osteoarthritis development $[5,6]$. In an HCS mouse model, overactive Notch2 signaling was found to trigger articular degeneration resembling osteoarthritis $(\mathrm{OA})$ [6].

In this case study, we present a HCS patient with EOOP and posttraumatic knee OA. The objectives were to demonstrate the feasibility of total knee arthroplasty (TKA) in HCS and to perform a multiscale, histomorphometric, skeletal characterization (including cartilage status), as there are still no corresponding detailed data for this rare skeletal disease. We also aimed to discuss the clinical orthopaedic management in patients with hereditary low bone mass disorders based on the case of HCS.

\section{Patient and methods}

We here describe a 38-year-old male patient with HajduCheney syndrome (HCS), who was diagnosed with HCS at the age of 13 years based on radiographic examination of the left hand demonstrating pathognomonic acroosteolysis and 
carpal dysplasia in synopsis with short stature, bathrocephaly, and facial dysmorphism (comprising a long philtrum, micrognathia, low-set ears, and hypertelorism) (Fig. 1a). In 2015, molecular genetic analysis for skeletal disorders revealed a frameshift mutation c.6657delC (p.Pro2219fs) of exon 34 of the NOTCH2 gene, located on chromosome 1p13$\mathrm{p} 11$, confirming the diagnosis of HCS as previously reported [7]. Due to manifest EOOP based on a severely reduced bone mineral density (BMD) and osteoporotic fractures of vertebrae Th8, Th9, L1, and L2, antiresorptive therapy with ibandronate had been initiated externally in 2011, which had been discontinued by the patient in 2012. After years without bone-specific therapy, alendronate was initiated externally in 2018. The patient suffered from progressive acroosteolysis of both hands, which was not positively affected by antiresorptive treatment (Fig. 1b). Furthermore, he had undergone numerous surgical procedures on both hands (i.e., bilateral radiocarpal arthrodesis and amputation of the right middle finger), as well as extensive oral and maxillofacial surgery (i.e., mandibular osteotomy, maxillary and mandibular
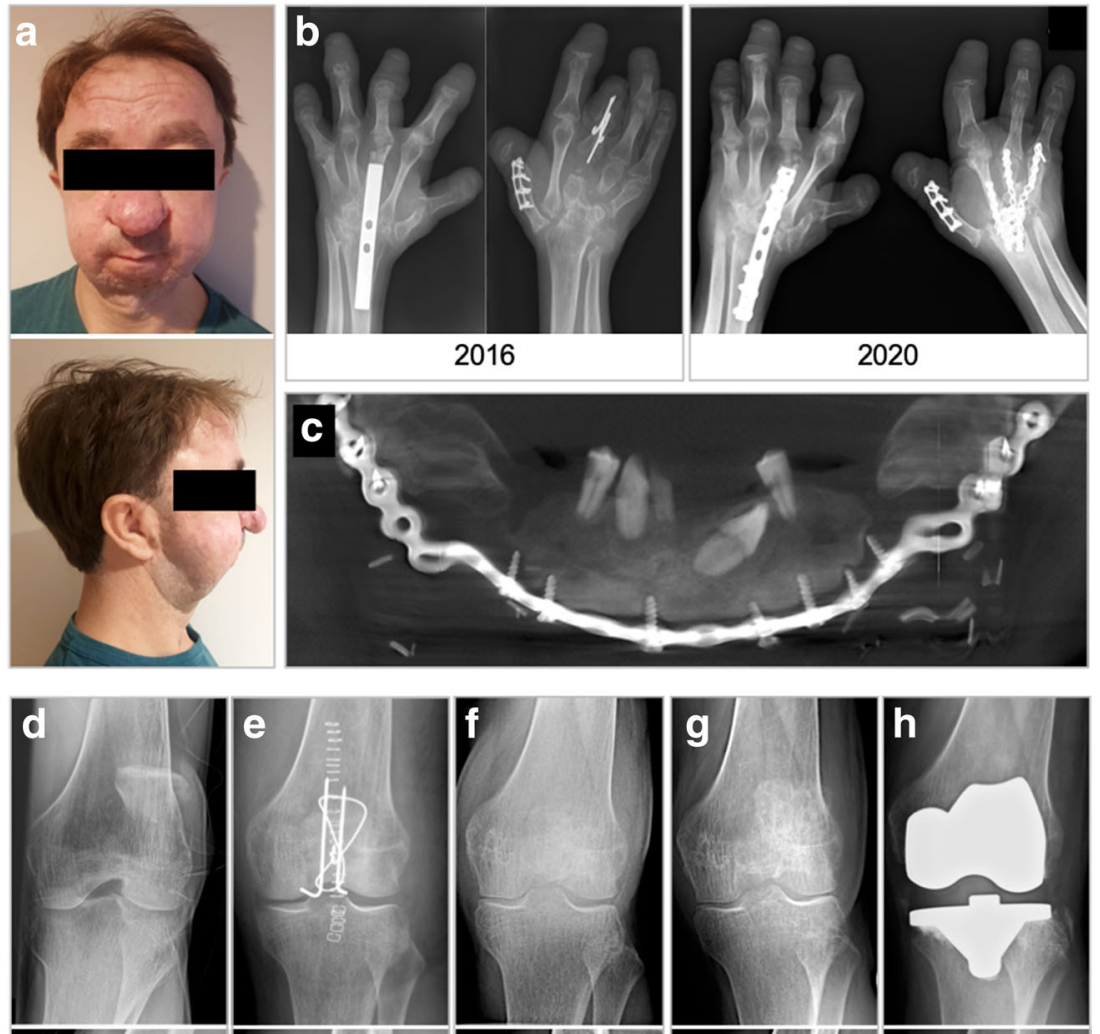

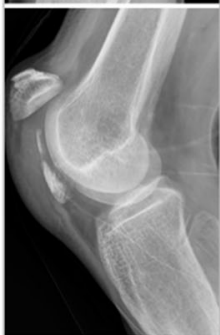

$09 / 2016$

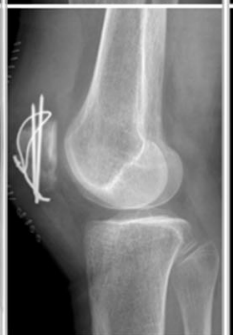

$09 / 2016$

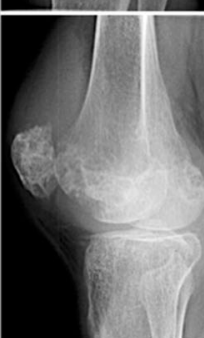

$12 / 2019$

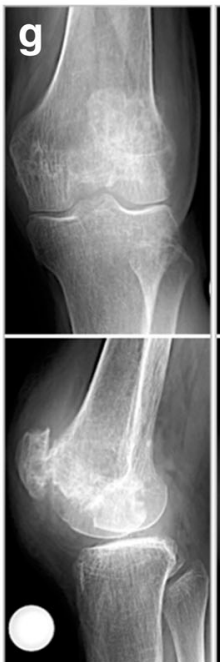

$08 / 2020$

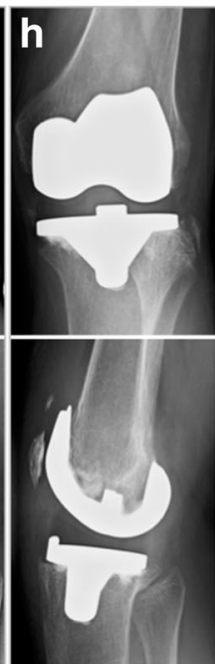

$01 / 2021$
Fig. 1 Clinical presentation and diagnostic imaging. a Facial dysmorphism comprised a long philtrum, micrognathia, premature loss of teeth, low-set ears, and hypertelorism, and caused the patient to undergo extensive oral and maxillofacial surgery (i.e., mandibular osteotomy, maxillary and mandibular prosthesis after multiple tooth extractions). b After experiencing atraumatic pain of the left hand during childhood, radiographic examination raised the suspicion of HCS, demonstrating pathognomonic acroosteolysis and carpal dysplasia. Acroosteolysis slightly progressed without noticeable positive effects under intermittent bisphosphonate treatment. Furthermore, he received numerous surgical procedures on both hands (i.e., bilateral radiocarpal arthrodesis and amputation of the right middle finger). $\mathbf{c}$ Cone-beam $\mathrm{CT}$ of the mandibula demonstrating the previous osteotomy with plate osteosynthesis as well as poor tooth status. $\mathbf{d}$ Between 2016 and 2020, the patient experienced a left-sided displaced comminuted patella fracture, $\mathbf{e}$ received tension band wiring and realignment surgery of the patella, $\mathbf{f}$ and removal of the cerclage due to mechanical complications. g Further on, he developed severe posttraumatic $\mathrm{OA}, \mathbf{h}$ finally leading to cemented implantation of a ligament-balanced total knee prosthesis (radiograph obtained 3 months postoperatively) 
prosthesis and multiple tooth extractions) (Fig. 1c). In 2016, after experiencing a displaced comminuted patella fracture (Fig. 1d) due to falling at ground level, the patient underwent tension band wiring and realignment surgery of the left patella (Fig. 1e). The cerclage wire was removed in 2018 due to mechanical complications (Fig. 1f). At presentation at our surgical department in 2020 after years of frustrating conservative therapy, diagnostic imaging revealed severe posttraumatic OA (Kellgren-Lawrence score: grade 4) of the knee with patellar deformity (Fig. 1g). He complained about chronic immobilizing pain, limping gait, and recurrent joint effusions, significantly restricting daily activities. On clinical examination, the knee presented with a moderately restricted range of motion/ROM (extension/flexion: $0-0-110^{\circ}$ ) with painful passive movement, and an intact ligamentous apparatus. Along the patient's decision for elective surgery, ligament-balanced TKA was performed using the Mathys ${ }^{\circledR}$ knee system balanSys BICONDYLAR (femur size C cemented, tibia size 70 cemented, polyethylene inlay fix UC 70/13, patella 3 peg flat 28 cemented) (Fig. 1h).

A detailed skeletal characterization of our patient comprised the assessment of BMD by dual-energy X-ray absorptiometry (DXA; Lunar iDXA, GE Healthcare, Madison, WI, USA) at both the lumbar spine and proximal femora, as well as high-resolution peripheral quantitative computed tomography (HR-pQCT; XtremeCT, Scanco Medical, Brütisellen, Switzerland) at the distal tibia. Laboratory bone metabolism markers included calcium, phosphate, cholecalciferol, parathyroid hormone, osteocalcin, bone-specific alkaline phosphatase, alkaline phosphatase, and urinary deoxypyridinoline. Furthermore, tibial cut sections were obtained intraoperatively from the HCS patient and a 46-year-old male control patient. This control patient suffered from advanced-stage varus OA and had undergone partial meniscectomy several years earlier with otherwise normal skeletal status. Histomorphometric analysis was carried out using the OsteoMeasure histomorphometry system (Osteometrics Inc., USA). The patients' clinical outcome was assessed by an age-appropriate questionnaire, quantifying the Knee Injury and Osteoarthritis Outcome Score (KOOS).

\section{Results}

Before and after surgery, extensive skeletal diagnostics were performed at our outpatient clinic, specialized in skeletal disorders (the National Bone Board, NBB). Between the initial presentation in 2014 and follow-up examination in 2020 (including 2 years of alendronate treatment), a mild increase in spinal BMD (T-score 2014: -5.2 ; T-score 2020: -4.8 ) but no changes in femoral BMD (T-score 2014: - 3.3; T-score 2020: - 3.3) were detected (Supp. Tab. 1). During this six-year period, the patient did not present to us and the recommendation of antiresorptive therapy escalation with denosumab due to pronounced and persistent increase in bone resorption under bisphosphonates was not implemented. He showed overall severely reduced trabecular and cortical microstructural parameters in 2014, with progressive microstructural deterioration by 2020 (Supp. Tab. 1 and Fig. 2a). In a detailed laboratory analysis of bone metabolism, we detected a persistent high bone turnover state (primarily high bone resorption) despite intermittent antiresorptive treatment and successful normalization of calcium metabolism by vitamin D supplementation of 20,000 IU per week (Fig. 2b).

During TKA, intraoperative cut sections of the tibia plateaus of the HCS patient (Fig. 2c) and the OA control patient were obtained for further analysis (Fig. 2d). Histology revealed severe articular cartilage degeneration and subchondral hyperosteoidosis. Detailed histomorphometry confirmed high osteoid levels as well as excessive osteoblast and osteoclast surfaces (Fig. 2e), indicative of increased subchondral bone turnover.

Following rehabilitation at 4 weeks after TKA, the HCS patient showed a regular improvement regaining mobility intermittently on elbow crutches. At 3-month follow-up, he presented a satisfactory result with a mostly pain-free physiologic ROM (extension/flexion: $0-0-130^{\circ}$ ), as well as great improvement of the KOOS profile (Fig. 2f). Simultaneously, radiographic imaging showed no signs of prosthetic loosening.

\section{Discussion}

This is the first available case report demonstrating the feasibility of TKA in a patient with HCS and severe OA of the knee, explaining challenges and perioperative strategies for the orthopedic surgeon. Following arthroplasty, loss of periprosthetic bone stock is seen in the general nonosteoporotic population $[8,9]$, and especially patients with genetic low bone mass diseases (e.g., HCS) present a distinct high-risk group concerning surgical challenges $[10,11]$ and adverse events (i.e., periprosthetic fractures or osteolysis with implant migration and prosthetic failure) [12]. In the field of orthopedic surgery, emerging developments highlight the importance of bone health optimization in patients with skeletal disorders, and a holistic therapeutic approach appears beneficial to optimize postoperative outcome [13].

Growing evidence supports the use of bisphosphonates in HCS patients [14]. Furthermore, good efficacy of the highly potent monoclonal anti-RANKL-antibody denosumab had been previously described in a patient case [15]. The rationale for continuation of antiresorptive therapy in patients with low BMD prior to elective endoprosthetic procedures is the preservation of periprosthetic BMD [9], most likely leading to improved prosthetic osseointegration and implant survival as well as postoperative fracture prevention [12]. In our HCS 
a
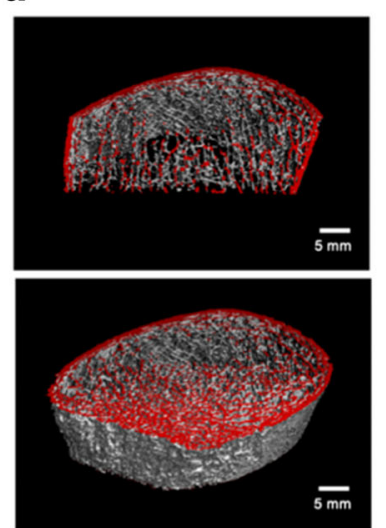

b
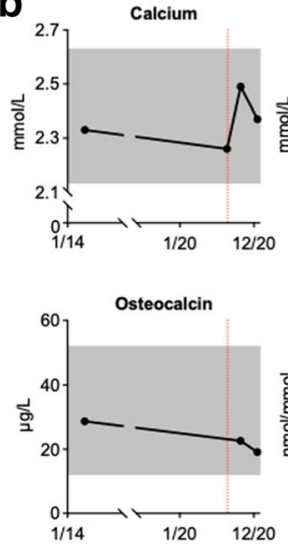
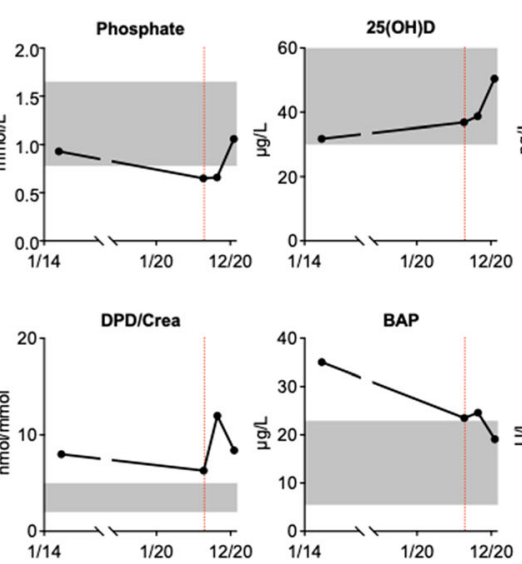
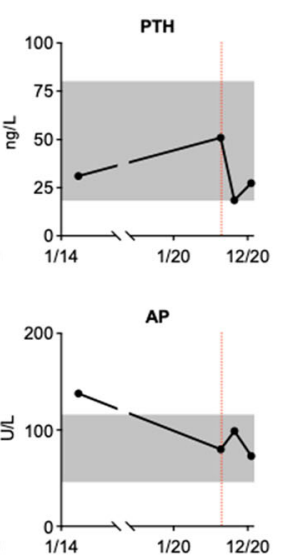

C

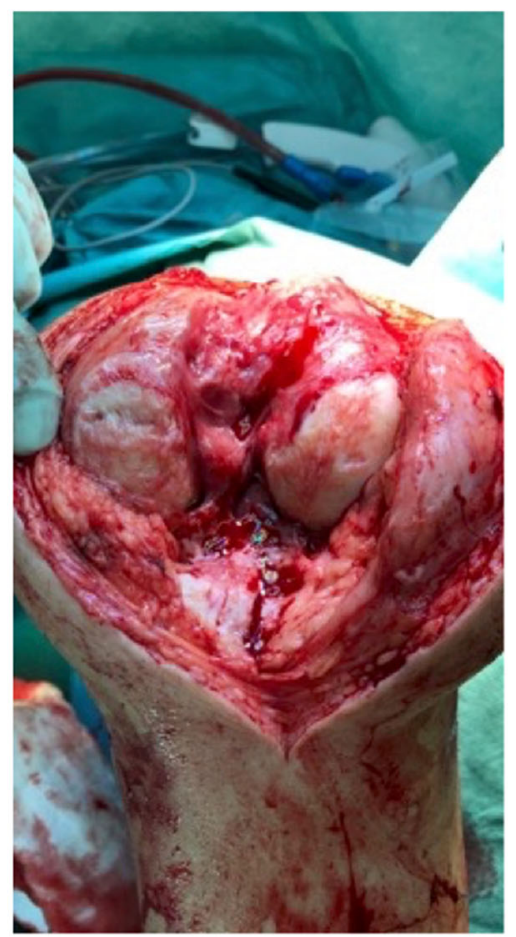

e

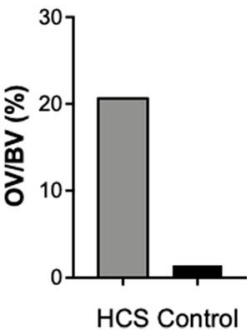

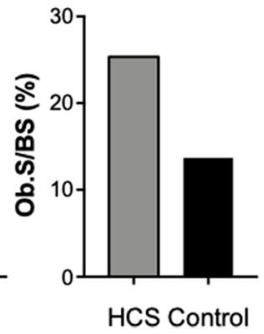

d

d HCS
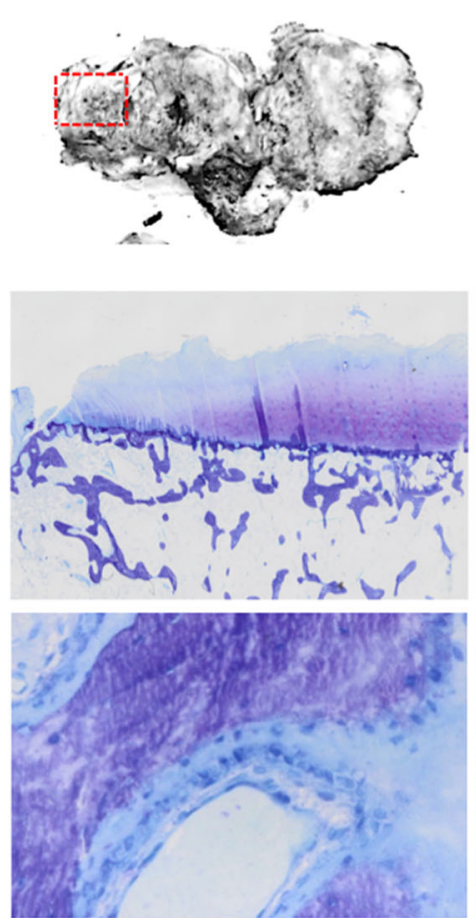

f

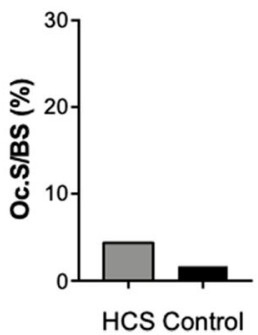

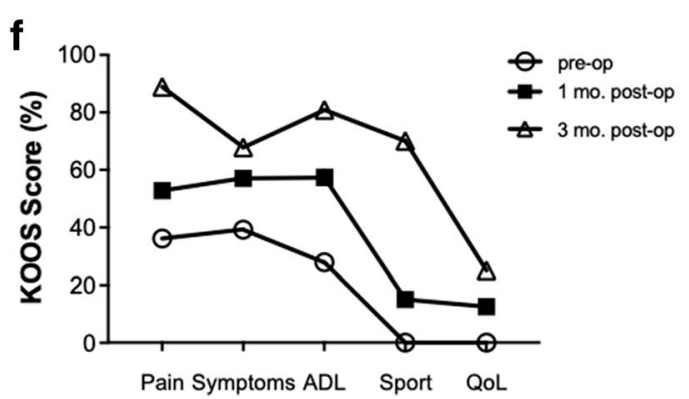

patient, the compromised bone status theoretically jeopardizes prosthetic osseointegration, substantiating cementation of the prosthesis at young age. In terms of preventive measures of peri-implant bone loss for patients undergoing TKA, moderate physical exercise can be recommended, as lean mass positively modulates periprosthetic BMD [16]. Finally, a close clinical and radiological follow-up should always be pursued in risk group patients undergoing orthopaedic surgical procedures.

This report provides a comprehensive and representative insight on the skeletal status in HCS, demonstrating the underlying high bone turnover, low bone stock, and the 
Fig. 2 Skeletal assessment and clinical outcome of TKA. a Between 2014 and 2020, comprising 2 years of alendronate treatment, peripheral bone microarchitecture assessed by HR-pQCT progressively deteriorated. b Laboratory control comprising serum (calcium, phosphate, cholecalciferol/25-OH-D3, parathyroid hormone/PTH, osteocalcin, bonespecific alkaline phosphatase/BAP, and alkaline phosphatase/AP) and urinary (deoxypyridinoline crosslinks per creatinine or DPD/Crea) bone metabolism parameters revealed high bone turnover at baseline with expected postoperative acceleration. The red-dotted line marks the date of TKA. $\mathbf{c}$ Intraoperative photograph of the affected knee joint, demonstrating the medially accentuated chondromalacia and a fibrosis-covered trochlea. $\mathbf{d}$ Macroscopic axial view on the intraoperatively obtained tibial plateaus of the patient and control (above), indicating the medial tibial resection specimens (red dotted rectangles). Histological overview (5X magnification; middle) and 20X magnification (below) of the osteochondral resection specimens, subjected to undecalcified histology and stained with toluidine blue. Comparing the HCS patient versus control, advanced cartilage degeneration as well as a large amount of osteoid were noted. e Histomorphometric quantification of osteoid volume per tissue volume (OV/BV), osteoblast surface per bone surface (Ob.S/BS), and osteoclast surface per bone surface (Oc.S/BS). e Clinical status (preoperative and postoperative) in the HCS patient, quantified by the Knee Injury and Osteoarthritis Outcome Score (KOOS), progressively improved in all five KOOS subscales

eterioration of microstructural parameters under intermittent antiresorptive therapy. Although histomorphometric findings are limited to a single case-control study design, they are in line with the scarce literature on histologic studies in human patients $[14,17,18]$ and the majority of findings in previous animal studies $[7,19,20]$. Opposed to our findings, solely the HCS mouse model by Canalis et al. [19] demonstrated decreased bone formation, questioning the high-turnover phenomenon and suggesting uncoupling of the skeletal metabolic processes in the case of HCS. Whether Notch2 exerts further osteolytic effects specifically on periprosthetic bone remains uncertain and subject to future research.

Next to the skeletal manifestations, this patient case underlines that OA may be a relevant complication of HCS. Our data confirm previous mouse model findings that have demonstrated potential joint sensitization to OA in Hajdu-Cheney mutant mice [6]. To allow quantification of a joint compartment that was affected as little as possible by the previous patella fracture, the articular cartilage from the medial tibia plateau was histologically analyzed. Although this analysis revealed advanced cartilage degeneration in the medial joint compartment and OA was radiologically present in all joint compartments (medial, lateral, and patellofemoral), it remains unclear to what extent the previous patella fracture affected OA development within the knee.

Detrimental effects on articular cartilage and subchondral bone might be related to the poorly understood pathophysiology of acroosteolysis, which involves focal inflammation with neovascularization/-innervation, mast cell infiltration, and fibrosis $[1,18,21]$. In this regard, it is possible that Notch2 hyperactivity exhibits complex systemic but site-specific mechanosensitive effects within the musculoskeletal system and links accelerated osteoarthritic and osteoporotic changes at the distal phalanges.

In conclusion, this is the first case of HCS in which TKA has been demonstrated as a successful treatment modality in order to address the patient's clinical impairment due to severe OA. We highlight the importance of adequate preoperative bone health optimization and present particular challenges to the orthopaedic surgeon. Moreover, our data provide an accurate skeletal characterization of HCS confirming previous mouse-model findings in terms of poor bone mass and advanced secondary cartilage loss. Since the observed severe $\mathrm{OA}$ in our patient may be related to the previous patella fracture, it has to be elucidated whether OA represents a relevant comorbidity in HCS patients independent of previous trauma.

Supplementary Information The online version contains supplementary material available at https://doi.org/10.1007/s00198-021-05914-6.

Funding Open Access funding enabled and organized by Projekt DEAL.

\section{Declarations}

Ethical approval No additional ethics approval for this case report is required.

Informed consent The authors declare that informed consent was obtained from the patient.

\section{Conflicts of interest None.}

Open Access This article is licensed under a Creative Commons Attribution-NonCommercial 4.0 International License, which permits any non-commercial use, sharing, adaptation, distribution and reproduction in any medium or format, as long as you give appropriate credit to the original author(s) and the source, provide a link to the Creative Commons licence, and indicate if changes were made. The images or other third party material in this article are included in the article's Creative Commons licence, unless indicated otherwise in a credit line to the material. If material is not included in the article's Creative Commons licence and your intended use is not permitted by statutory regulation or exceeds the permitted use, you will need to obtain permission directly from the copyright holder. To view a copy of this licence, visit http:// creativecommons.org/licenses/by-nc/4.0/.

\section{References}

1. Canalis E, Zanotti S (2014) Hajdu-Cheney syndrome: a review. Orphanet J Rare Dis 9:1-7

2. Simpson MA, Irving MD, Asilmaz E, Gray MJ, Dafou D, Elmslie FV, Mansour S, Holder SE, Brain CE, Burton BK, Kim KH, Pauli RM, Aftimos S, Stewart H, Kim CA, Holder-Espinasse M, Robertson SP, Drake WM, Trembath RC (2011) Mutations in 
NOTCH2 cause Hajdu-Cheney syndrome, a disorder of severe and progressive bone loss. Nat Genet 43:303-305

3. Zanotti S, Canalis E (2016) Notch signaling and the skeleton. Endocr Rev 37:223-253

4. Monteagudo S, Lories RJ (2018) A Notch in the joint that exacerbates osteoarthritis. Nat Rev Rheumatol 14:563-564

5. Hosaka Y, Saito T, Sugita S, Hikata T, Kobayashi H, Fukai A, Taniguchi Y, Hirata M, Akiyama H, Chung UI, Kawaguchi H (2013) Notch signaling in chondrocytes modulates endochondral ossification and osteoarthritis development. Proc Natl Acad Sci U S A 110:1875-1880

6. Zanotti S, Yu J, Bridgewater D, Wolf JM, Canalis E (2018) Mice harboring a Hajdu-Cheney syndrome mutation are sensitized to osteoarthritis. Bone 114:198-205

7. Vollersen N, Hermans-Borgmeyer I, Cornils K, Fehse B, Rolvien T, Triviai I, Jeschke A, Oheim R, Amling M, Schinke T, Yorgan TA (2018) High bone turnover in mice carrying a pathogenic Notch2 mutation causing Hajdu-Cheney syndrome. J Bone Miner Res 33:70-83

8. Blaty T, Krueger D, Illgen R, Squire M, Heiderscheit B, Binkley N, Anderson P (2019) DXA evaluation of femoral bone mineral density and cortical width in patients with prior total knee arthroplasty. Osteoporos Int 30:383-390

9. Shi M, Chen L, Xin Z, Wang Y, Wang W, Yan S (2018) Bisphosphonates for the preservation of periprosthetic bone mineral density after total joint arthroplasty: a meta-analysis of 25 randomized controlled trials. Osteoporos Int 29:1525-1537

10. Mattei TA, Rehman AA, Issawi A, Fassett DR (2015) Surgical challenges in the management of cervical kyphotic deformity in patients with severe osteoporosis: an illustrative case of a patient with Hajdu-Cheney syndrome. Eur Spine J 24:2746-2753

11. Dokou P, Karoussis IK, Papavasiliou G, Kamposiora P, Vrahopoulos TP, Vrotsos JA (2016) Osseointegration of dental implants in a patient with Hajdu-Cheney syndrome. Open Dent J 10:575-586

12. Russell LA (2013) Osteoporosis and orthopedic surgery: effect of bone health on total joint arthroplasty outcome. Curr Rheumatol Rep 15:371
13. Anderson PA, Jeray KJ, Lane JM, Binkley NC (2019) Bone health optimization: Beyond own the bone: AOA critical issues. J Bone Jt Surg - Am Vol 101:1413-1419

14. Sakka S, Gafni RI, Davies JH, Clarke B, Tebben P, Samuels M, Saraff V, Klaushofer K, Fratzl-Zelman N, Roschger P, Rauch F, Högler W (2017) Bone structural characteristics and response to bisphosphonate treatment in children with Hajdu-Cheney syndrome. J Clin Endocrinol Metab 102:4163-4172

15. Adami G, Rossini M, Gatti D, Orsolini G, Idolazzi L, Viapiana O, Scarpa A, Canalis E (2016) Hajdu-Cheney syndrome; report of a novel NOTCH2 mutation and treatment with denosumab. Bone 92: $150-156$

16. Mau-Moeller A, Behrens M, Felser S, Bruhn S, Mittelmeier W, Bader R, Skripitz R (2015) Modulation and predictors of periprosthetic bone mineral density following total knee arthroplasty. Biomed Res Int 2015:418168

17. Leidig-Bruckner G, Pfeilschifter J, Penning N, Limberg B, Priemel M, Delling G, Ziegler R (1999) Severe osteoporosis in familial Hajdu-Cheney syndrome: progression of acro-osteolysis and osteoporosis during long-term follow-up. J Bone Miner Res 14:20362041

18. Udell J, Schumacher HR Jr, Kaplan F, Fallon MD (1986) Idiopathic familial acroosteolysis: histomorphometric study of bone and literature review of the Hajdu-Cheney syndrome. Arthritis Rheum 8: 1032-1038

19. Canalis E, Schilling L, Yee SP, Lee SK, Zanotti S (2016) HajduCheney mouse mutants exhibit osteopenia, increased osteoclastogenesis, and bone resorption. J Biol Chem 291:1538-1551

20. Zanotti S, Yu J, Sanjay A, Schilling L, Schoenherr C, Economides AN, Canalis E (2017) Sustained Notch2 signaling in osteoblasts, but not in osteoclasts, is linked to osteopenia in a mouse model of Hajdu-Cheney syndrome. J Biol Chem 292:12232-12244

21. Mapp PI, Walsh DA (2012) Mechanisms and targets of angiogenesis and nerve growth in osteoarthritis. Nat Rev Rheumatol 8:390398

Publisher's note Springer Nature remains neutral with regard to jurisdictional claims in published maps and institutional affiliations. 